\title{
Spin and charge order in doped spin-orbit coupled Mott insulators
}

\author{
Mehdi Biderang,,${ }^{1,2, *}$ Alireza Akbari, ${ }^{3,4, \dagger}$ and Jesko Sirker ${ }^{1,2, \ddagger}$ \\ ${ }^{1}$ Department of Physics and Astronomy, University of Manitoba, Winnipeg R3T 2N2, Canada \\ ${ }^{2}$ Manitoba Quantum Institute, University of Manitoba, Winnipeg R3T 2N2, Canada \\ ${ }^{3}$ Max Planck Institute for the Chemical Physics of Solids, D-01187 Dresden, Germany \\ ${ }^{4}$ Max Planck POSTECH Center for Complex Phase Materials, \\ and Department of Physics, POSTECH, Pohang, Gyeongbuk 790-784, Korea
}

(Dated: December 22, 2020)

\begin{abstract}
We study a two-dimensional single band Hubbard Hamiltonian with antisymmetric spin-orbit coupling. We argue that this is the minimal model to understand the electronic properties of locally non-centrosymmetric transition-metal (TM) oxides such as $\mathrm{Sr}_{2} \mathrm{IrO}_{4}$. Based on exact diagonalizations of small clusters and the random phase approximation, we investigate the correlation effects on charge and magnetic order as a function of doping and of the TM-oxygen-TM bond angle $\theta$. For small doping and $\theta \lesssim 15^{\circ}$ we find dominant commensurate inplane antiferromagnetic fluctuations while ferromagnetic fluctuations dominate for $\theta \gtrsim 25^{\circ}$. Moderately strong nearest-neighbor Hubbard interactions can also stabilize a charge density wave order. Furthermore, we compare the dispersion of magnetic excitations for the hole-doped case to resonant inelastic X-ray scattering data and find good qualitative agreement.
\end{abstract}

\section{INTRODUCTION}

Solid state systems with strong electron correlations show an intriguing number of quantum many-body phenomena including high-temperature superconductivity, spinliquid phases, colossal magnetoresistance, and multiferroic behavior. They can also host exotic quasiparticles such as Majorana and Weyl fermions [1-11]. Another recurring theme in contemporary condensed matter physics is the emergence of various types of charge and spin order in strongly interacting systems [12, 13]. Interesting ordering phenomena were experimentally observed in many rare-earth and transition metal oxide (TMO) compounds, for example, $\mathrm{La}_{1.6} \mathrm{Nd}_{0.4} \mathrm{Sr}_{x} \mathrm{CuO}_{4}, \mathrm{YBa}_{2} \mathrm{Cu}_{3} \mathrm{O}_{6+x}, \mathrm{Bi}_{2} \mathrm{Sr}_{2} \mathrm{Cu}_{2} \mathrm{O}_{8+x}$, $\mathrm{La}_{1.5} \mathrm{Sr}_{0.5} \mathrm{NiO}_{4}, \mathrm{Na}_{x} \mathrm{CoO}_{2}$, and $\mathrm{Sr}_{2} \mathrm{IrO}_{4}$ some of which also exhibit high temperature superconductivity [14-18]. Strong electron interactions make TMOs a particularly promising class of materials to find novel exotic phases [3, 6].

In 5d TMOs, the presence of crystal fields, spin-orbit couplings (SOC), and strong Coulomb interactions leads to enhanced quantum fluctuations and a competition between a variety of often exotic ground states [19-21]. Among these materials, the iridates and especially the layered perovskite $\mathrm{Sr}_{2} \mathrm{IrO}_{4}$ has attracted a lot of attention due to its similarities with the cuprate superconductors [22-29]. For example, $\mathrm{Sr}_{2} \mathrm{IrO}_{4}\left(\mathrm{La}_{2} \mathrm{CuO}_{4}\right)$ has one hole per $\mathrm{Ir}(\mathrm{Cu})$ ion, and shows a pseudospin- $\frac{1}{2}$ antiferromagnetic order. Moreover, recent experiments on electron-doped $\mathrm{Sr}_{2} \mathrm{IrO}_{4}$ indicate the emergence of a pseudogap and, at low temperatures, of a d-wave gap which strengthens the analogy with the cuprates [8]. On the other hand, there are also distinct differences. $\mathrm{Sr}_{2} \mathrm{IrO}_{4}$ has, in particular, large spin-orbit couplings and a non-symmorphic crystal structure.

\footnotetext{
*mehdi.biderang@umanitoba.ca

† akbari@postech.ac.kr

‡sirker@physics.umanitoba.ca
}

Triggered by the discovery of superconductivity in the non-centrosymmetric (NCS) superconductor $\mathrm{CePt}_{3} \mathrm{Si}$, the role played by antisymmetric spin-orbit coupling (ASOC) for the electronic and topological properties of a band structure has come into focus [30-40]. One of the most intriguing features of topological non-centrosymmetric superconductors is that they can host Majorana fermions [41, 42]. Locally NCS superconductors belong globally to a centrosymmetric space group (global inversion symmetry) [43], however, as a result of randomly distributed stacking faults the inversion symmetry is locally broken $[44,45]$. Compounds belonging to this class are, for example, $\mathrm{Sr}_{3} \mathrm{Ru}_{2} \mathrm{O}_{7}, \mathrm{Sr}_{2} \mathrm{RhO}_{4}$, and $\mathrm{Sr}_{2} \mathrm{IrO}_{4}[46,47]$. Here distortions in the TMO-oxygen-TMO bonds break inversion symmetry locally and lead to staggered ASOCs.

The crystal structure of $\mathrm{Sr}_{2} \mathrm{IrO}_{4}$ has been investigated experimentally in a number of studies. Early neutron powder diffraction measurements indicated that the crystal structure of $\mathrm{Sr}_{2} \mathrm{IrO}_{4}$ belongs to the centrosymmetric space group $I 4_{1} /$ acd $[48,49]$. More recent studies by single-crystal neutron diffraction revealed, however, a $I 4_{1} / a$ space group $[50,51]$. In both cases, the crystal structure is globally centrosymmetric and non-symmorphic. Since local inversion symmetry at the Ir sites is missing, ASOC and the entanglement of various internal degrees of freedom are expected to occur in this locally NCS system [44, 45]. Moreover, the results of resonant inelastic X-ray scattering (RIXS) on electron-doped $\mathrm{Sr}_{2} \mathrm{IrO}_{4}$ show that magnetic correlations persist well into the metallic regime while the long-range magnetic order is lost [52-54]. This property is another similarity to the case of hole-doped cuprates. I.e., there is a type of electron-hole conjugation between the properties of the iridates and those of the cuprates [23].

The problem of the interplay between spin-orbit interactions, magnetic and charge fluctuations has been considered for a broad range of strongly correlated electron materials, such as cuprate high- $T_{c}$ superconductors, heavy fermion compounds, and TMOs [55]. In the particular case of the cuprates, the role of charge and antiferromagnetic spin fluctuations and 


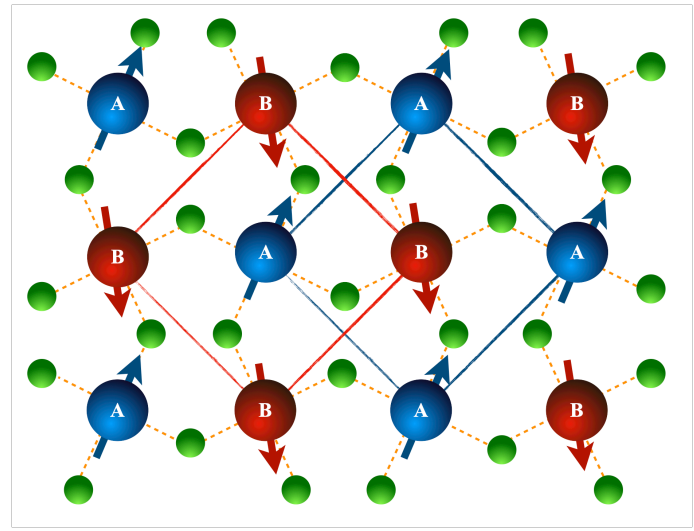

FIG. 1. The unit cell of the layered perovskite $\mathrm{Sr}_{2} \mathrm{IrO}_{4}$. The red and blue circles denote Ir atoms at different sublattices with the letters A and $\mathrm{B}$. The red and purple arrows indicate the magnetic moments on Ir sites. The green circles are oxygen atoms. The deviation of Ir-O-Ir bonds from $180^{\circ}$ breaks the local inversion symmetry and generates a non-colinear antiferromagnetic order along with a partially ferromagnetic moment.

their relation to superconductivity remains controversial. In this paper, we will investigate the charge and magnetic properties of a two-dimensional single band Hubbard model with antisymmetric spin-orbit coupling describing materials such as the $5 d$ layered $\mathrm{TMO} \mathrm{Sr}_{2} \mathrm{IrO}_{4}$. Our model is taking into account a next-nearest neighbor hopping, which leads to an asymmetry of hole and electron doping, as well as nearestneighbor Hubbard interactions which can lead to charge order. In addition, the effects of the rotation of the $\mathrm{IrO}_{6}$ octahedra are included. Our main goal is to investigate the dominant spin and charge fluctuations across the phase diagram of this model. Apart from being of interest in their own right, this study will also set the stage to discuss the mechanisms for superconductivity in future studies.

The paper is organized as follows: In Sec. II we introduce the model and consider its fundamental properties in the noninteracting case. In Sec. III we then use exact diagonalizations of small clusters to develop some understanding of the dominant short-range magnetic fluctuations in the interacting case. Next, we derive in Sec. IV the dynamical charge and spin susceptibilities as well as the magnon dispersions using the random-phase approximation (RPA). The last section is devoted to a short summary and conclusion.

\section{DESCRIPTION OF THE MODEL}

$\mathrm{Sr}_{2} \mathrm{IrO}_{4}$ is a layered material based on the stacking of two-dimensional $\mathrm{IrO}_{2}$ sheets, in which $\mathrm{Ir}^{4+}$ ions form a square lattice with two sublattices. At each lattice point, the $\mathrm{IrO}_{6}$ octahedron is elongated along the c-axis and rotated around it by an angle of $\theta \approx 11^{\circ}$, leading to a locally broken inversion symmetry at each sublattice. X-ray scattering and neutron diffraction experiments detected this local non-centrosymmetricity as a canted antiferromagnetic order $[56,57]$. Besides, measurements of the magnetic suscepti- bility revealed a weak ferromagnetic moment [58]. The unit cell of $\mathrm{Sr}_{2} \mathrm{IrO}_{4}$ and the magnetic order is shown schematically in Fig. 1.

A sufficiently large crystal field in a $5 d$ TMO splits the $5 d$ orbitals into a $t_{2 g}$ triplet and an $e_{g}$ doublet. The $t_{2 g}$ states are the low-spin ground states of the system [59, 60]. For strong SOC, the $t_{2 g}$ orbitals - an effective $L=1$ systems - is split further into fully filled $J_{\text {eff }}=3 / 2$ and half-filled $J_{\text {eff }}=1 / 2$ (upper) states $[59,60]$. The reduction of the bandwidth due to SOC causes the formation of a Mott-insulating ground state in the presence of an intermediate amount of correlation between the electrons [61]. The single-band Mott-insulating picture for the low-energy physics of Ir oxides with $J_{\text {eff }}=1 / 2$ appears to be consistent with a number of experimental and theoretical investigations. Based on this simplified single band picture, the Hubbard-type model which we want to investigate is given by

$$
\mathcal{H}=\mathcal{H}_{0}+\mathcal{H}_{\text {Int }},
$$

where $\mathcal{H}_{0}$ and $\mathcal{H}_{\text {Int }}$ are the non-interacting and interacting parts of the Hamiltonian, respectively. The non-interacting part in real space can be written as

$$
\begin{aligned}
\mathcal{H}_{0}=-\mu & -\sum_{\langle i j\rangle, \sigma}\left\{t_{1}\left[a_{i \sigma}^{\dagger} b_{j \sigma}+b_{i \sigma}^{\dagger} a_{j \sigma}\right]\right. \\
& \left.+\sum_{\sigma^{\prime}} \mathrm{i} t_{1}^{\prime} \hat{\sigma}_{\sigma \sigma^{\prime}}^{z}\left[a_{i \sigma}^{\dagger} b_{j \sigma^{\prime}}-b_{i \sigma}^{\dagger} a_{j \sigma^{\prime}}\right]\right\} \\
& -\sum_{\langle\langle i j\rangle, \sigma}\left\{t_{2}\left[a_{i \sigma}^{\dagger} a_{j \sigma}+b_{i \sigma}^{\dagger} b_{j \sigma}\right]\right. \\
& \left.+\sum_{\sigma^{\prime}} \mathrm{i} t_{2}^{\prime}\left(\hat{\boldsymbol{\sigma}} \times \hat{\mathbf{r}}_{i j}\right)_{\sigma \sigma^{\prime}}^{z}\left[a_{i \sigma}^{\dagger} a_{j \sigma^{\prime}}-b_{i \sigma}^{\dagger} b_{j \sigma^{\prime}}\right]\right\},
\end{aligned}
$$

where $\hat{\boldsymbol{\sigma}}=\left(\sigma^{x}, \sigma^{y}, \sigma^{z}\right)$ denotes the $2 \times 2$ Pauli matrices in the pseudospin basis, $\mu$ is the chemical potential, and $a_{i \sigma}^{\dagger}\left(b_{i \sigma}^{\dagger}\right)$ creates an electron at site $i$ in sublattice A (B) with pseudospin $\sigma$. The parameter $t_{2}$ denotes the second neighbor spin-independent (intra-sublattice) hopping integral and $t_{1}=t_{1}(\theta)=\frac{2 t_{0}}{3} \cos \theta\left(2 \cos ^{4} \theta-1\right)$ and $t_{1}^{\prime}=$ $t_{1}^{\prime}(\theta)=\frac{2 t_{0}}{3} \sin \theta\left(2 \sin ^{4} \theta-1\right)$ are spin-independent and spindependent (inter-sublattice) nearest-neighbor hopping amplitudes, respectively [61]. Here, the angle $\theta$ describes the rotation of the $\mathrm{IrO}_{6}$ octahedra along the c-axis. The last term in the non-interacting Hamiltonian stems from the second neighbor spin-dependent (intra-sublattice) hopping with amplitude $t_{2}^{\prime}$, and is a staggered ASOC that violates parity. The combination of the $\mathrm{IrO}_{6}$ rotation with the stacking structure of the 2D layers along the c-axis breaks the mirror symmetry with regard to the ab-plane and results in the spin-dependent intrasublattice term [44]. Moreover, the crinkling of the lattice by displacing the A (B) sublattice in the $\hat{z}(-\hat{z})$ direction allows for a second-neighbor (intra-sublattice) spin-dependent hopping [62]. To obtain the previously reported electronic band structure [27, 61, 63, 64], we set the hopping parameters $t_{2}=t_{1}^{2}$ and $t_{2}^{\prime}=t_{1}^{\prime 2}$, respectively. Furthermore, all of the physical parameters are scaled in units of $t_{0}=0.35 \mathrm{eV}$ to obtain a band structure in good agreement with LDA+SOC calculations [27, 61, 63]. 

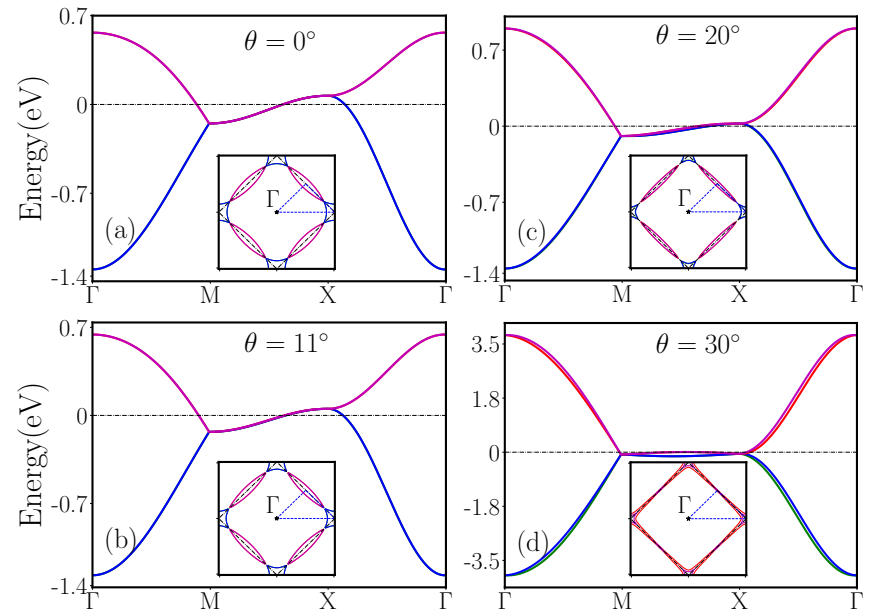

FIG. 2. Evolution of the non-interacting band structure (main plots) and the Fermi surface (insets) of the $J_{\text {eff }}=1 / 2$ model (2) with respect to changes in the canting angle for the electron-doped case with $\langle n\rangle=2.4$. The first BZ with the $\mathrm{A}$ and $\mathrm{B}$ sublattices is depicted as dashed lines. Increasing the distortion of the Ir-O-Ir bonds will reduce the second neighbor hopping and ultimately restore the particle-hole symmetry of the band structure.

The repulsive interactions in Eq. (1) are taken as a combination of both on-site and extended Hubbard terms

$$
\mathcal{H}_{\text {Int }}=\frac{U}{2} \sum_{i, \sigma} n_{i \sigma} n_{i \bar{\sigma}}+\frac{V}{2} \sum_{\langle i j\rangle, \sigma \sigma^{\prime}} n_{i \sigma} n_{j \sigma^{\prime}}
$$

where $U$ and $V$ are the strengths of the on-site and the firstneighbor Hubbard interaction, respectively, $n_{i \sigma}$ is the pseudospin dependent electron occupation number operator, and we set $\bar{\sigma}=-\sigma$.

Next, we perform a Fourier transformation of the fermionic operators $a_{\mathbf{k} \sigma}^{\dagger}=\frac{1}{N} \sum_{i} e^{\mathrm{i} \mathbf{k} \cdot \mathbf{R}_{i}} a_{i \sigma}^{\dagger}$, and $b_{\mathbf{k} \sigma}^{\dagger}=\frac{1}{N} \sum_{i} e^{\mathrm{i} \mathbf{k} \cdot \mathbf{R}_{i}} b_{i \sigma}^{\dagger}$ for the sublattices $A$ and $B$, respectively, with the number of points in momentum space denoted by $N$. This leads to the Hamiltonian in reciprocal space, which can be written as

$$
\mathcal{H}_{0}=\sum_{\mathbf{k}} \Psi_{\mathbf{k}}^{\dagger}\left[\begin{array}{cc}
\varepsilon_{2 \mathbf{k}}+\mathbf{g}_{2 \mathbf{k}} \cdot \boldsymbol{\sigma} & \varepsilon_{1 \mathbf{k}}+\mathrm{ig}_{1 \mathbf{k}} \cdot \boldsymbol{\sigma} \\
\varepsilon_{1 \mathbf{k}}-\mathrm{ig}_{1 \mathbf{k}} \cdot \boldsymbol{\sigma} & \varepsilon_{2 \mathbf{k}}-\mathbf{g}_{2 \mathbf{k}} \cdot \boldsymbol{\sigma}
\end{array}\right] \Psi_{\mathbf{k}}
$$

where $\Psi_{\mathbf{k}}^{\dagger}=\left(a_{\mathbf{k} \uparrow}^{\dagger}, a_{\mathbf{k} \downarrow}^{\dagger}, b_{\mathbf{k} \uparrow}^{\dagger}, b_{\mathbf{k} \downarrow}^{\dagger}\right)$, and

$$
\begin{aligned}
& \varepsilon_{1 \mathbf{k}}=-4 t_{1}\left[\cos k_{x}+\cos k_{y}\right], \\
& \varepsilon_{2 \mathbf{k}}=-4 t_{2} \cos k_{x} \cos k_{y}-\mu,
\end{aligned}
$$

are the dispersions originating from the nearest-neighbor (inter-sublattice) and next-nearest neighbor (intra-sublattice) spin-independent hopping, respectively. Furthermore,

$$
\mathbf{g}_{1 \mathbf{k}}=-4 t_{1}^{\prime}\left[\cos k_{x}+\cos k_{y}\right] \hat{z}
$$

corresponds to the nearest-neighbor (inter-sublattice) spindependent hopping. This term is a consequence of a deviation of the Ir-O-Ir bond angle from $180^{\circ}$ which generates a
quasi-SOC described by an even vector $\mathbf{g}_{1 \mathbf{k}}$. Moreover,

$$
\mathbf{g}_{2 \mathbf{k}}=-4 t_{2}^{\prime}\left[\sin k_{x} \cos k_{y} \hat{x}-\sin k_{y} \cos k_{x} \hat{y}\right],
$$

describes the ASOC g-vector. Eqs. (6) and (7) show that only the ASOC results in a violation of parity. The energy dispersion of the non-interacting normal system is therefore given by

$$
E_{\mathbf{k}, s}=-\mu+\varepsilon_{2 \mathbf{k}}+\xi \sqrt{\varepsilon_{1 \mathbf{k}}^{2}+\left(\mathbf{g}_{1 \mathbf{k}}+\xi^{\prime}\left|\mathbf{g}_{2 \mathbf{k}}\right|\right)^{2}},
$$

where $s=\{1,2,3,4\}$ denotes the band number corresponding to $\left(\xi, \xi^{\prime}\right)=\{(-1,-1),(-1,+1),(+1,-1),(+1,+1)\}$, respectively. The band filling is defined as the number of electrons per unit cell and expressed as $\langle n\rangle=2+2 \rho$, in which $\rho$ corresponds to the doping level, e.g. $\langle n\rangle=2$ for half-filling. It is worth mentioning that at every specific level of doping, the value of the chemical potential can be calculated from

$$
\rho=\frac{1}{N} \sum_{\mathbf{k}, s} f\left(E_{\mathbf{k}, s}\right)-2
$$

where $f(\ldots)$ the Fermi-Dirac distribution function at temperature $T$. We are interested here in the limit $T \rightarrow 0$.

Let us now discuss the evolution of the non-interacting band structure and the Fermi surface with respect to the rotation of the $\mathrm{IrO}_{6}$ octahedra around the $z$ axis at a specific filling of $\langle n\rangle=2.4$. Fig. 2(a) shows the case of canting angle $\theta=0^{\circ}$. In this case, the band structure and Fermi surface is two-fold degenerate due to the absence of both first- and second-neighbor spin-dependent hopping. Figs. 2(b-d) show the effect of an increase in the canting angle on the band structure and Fermi surface. In particular, the splitting between the bands increases with increasing angle $\theta$. Moreover, an increasing canting angle also reduces the amplitude of the next-nearest neighbor spin-independent hopping. This leads to almost particle-hole symmetric bands at the largest canting angle $\theta=30^{\circ}$ shown in Fig. 2(d). At the same time, semiflat bands and line nodes are formed along the Brillouin zone (BZ) boundary from the $\mathrm{M}$ to the $\mathrm{X}$ high-symmetry points. The corresponding evolution of the Fermi surface is shown in the insets of Fig. 2.

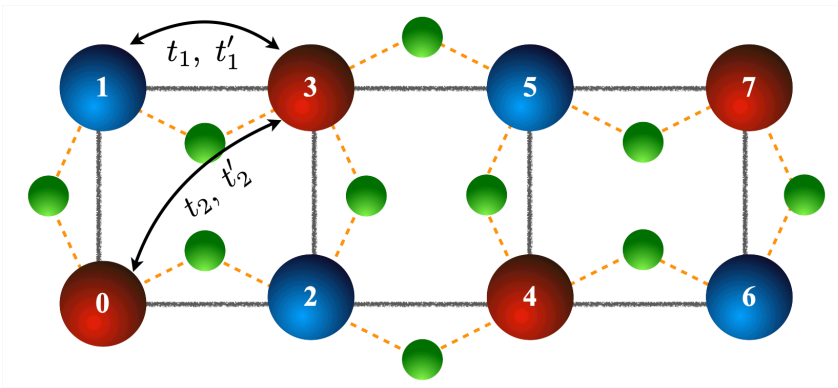

FIG. 3. The basic building block of our model is a two-leg ladder with intra-sublattice hopping amplitudes $t_{1}$ and $t_{1}^{\prime}$ and intersublattice hopping amplitudes $t_{2}$ and $t_{2}^{\prime}$. We consider an onsite Hubbard interaction $U$ and a nearest-neighbor interaction $V$. 

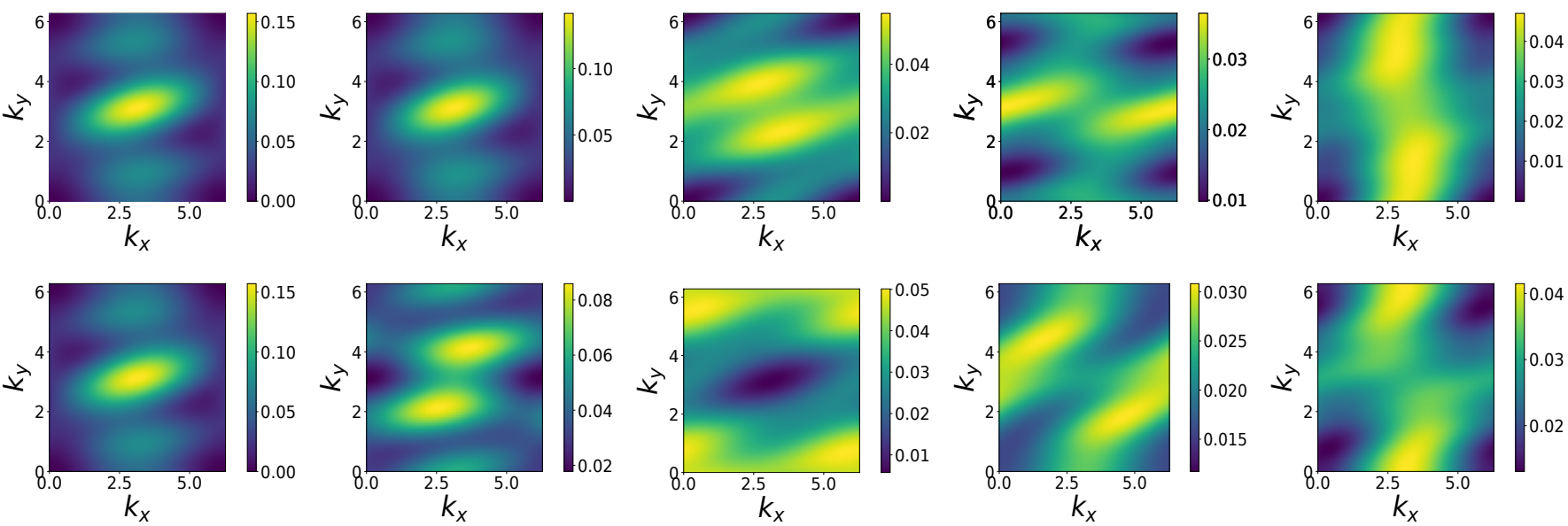

FIG. 4. Magnetic structure as obtained from exact diagonalizations of small clusters. In all cases $U=4$ and $V=0.4$. Upper row, left to right: static longitudinal spin structure factors for a $2 \times 4$ cluster with $\langle n\rangle=2$ and $\theta=0^{\circ}, 20^{\circ}, 30^{\circ}$ followed by the results for a $2 \times 5$ cluster with $\theta=20^{\circ}$ and $\langle n\rangle=2.8$ and finally $\langle n\rangle=1.2$. The lower row shows the corresponding transverse structure factors.

\section{EXACT RESULTS FOR SMALL CLUSTERS}

In order to gain some insights into the interplay of the various terms in Eq. (2) and the Coulomb interactions (3), we start by considering small clusters using exact diagonalizations. As the central building block, we consider a two-leg ladder of alternating A and B sublattice sites which constitutes 'two rows' in the lattice shown in Fig. 1. This two-leg ladder-including the various hopping terms - is also shown more schematically in Fig. 3. Because spin is not a good quantum number due to the $t_{2}^{\prime}$ term, we only have particle number conservation to limit the size of the Hilbert space thus restricting our exact diagonalizations to relatively small cluster sizes. Nevertheless, as we show below, these cluster sizes are sufficient to gain some valuable insights.

We concentrate on intermediate interaction strengths $U=4$ and $V=0.4$ and checked that the results are qualitatively very similar for $U=2$ to $U=8$ with $V=U / 10$. Furthermore, for $V \ll U$, as is expected for $\mathrm{Sr}_{2} \mathrm{IrO}_{4}$, there will be no charge order. We thus focus entirely on the magnetic order as a function of canting angle $\theta$ and doping level $\langle n\rangle$. We consider a two-leg ladder with open boundary conditions and calculate the correlation functions $\left\langle S_{0}^{z} S_{r}^{z}\right\rangle$ and $\left\langle S_{0}^{+} S_{r}^{-}\right\rangle$where the sites are numerated as shown in Fig. 3. A Fourier transform then leads to the static spin structure factors $S^{z z}(\mathbf{k})$ and $S^{+-}(\mathbf{k})$ which we use as our main observables to discuss the possible magnetic orderings. While dynamical structure factors can be calculated as well, we find that the accessible cluster sizes are too small to learn much about the dispersion of the magnetic excitations.

In Fig. 4, results for the static spin structure factors are shown for different canting angles and filling fractions. We start by considering the half-filled case, $\langle n\rangle=2$. For $\theta=0^{\circ}$, the model has $S U(2)$ spin rotational symmetry and the longitudinal and transverse spin correlations are identical. For this case we find strong antiferromagnetic correlations, see the leftmost column in Fig. 4. There is a peak in the structure factor centered at $(\pi, \pi)$. Increasing the canting angle in the half-filled case, the magnetic structure remains largely unchanged up to $\theta \lesssim 20^{\circ}$ although transverse and longitudinal spin correlators are, of course, no longer exactly equal. For the case $\theta=20^{\circ}$, shown in the second column of Fig. 4, $S^{+-}(\mathbf{k})$ shows a splitting of the antiferromagnetic peak into two incommensurate peaks. For $\theta=30^{\circ}$, shown in the third column of Fig. 4, most of the spectral weight in $S^{+-}(\mathbf{k})$ has moved to $\mathbf{k}=(0,0)$ (and points equivalent by a reciprocal lattice vector), i.e., the in-plane magnetic correlations are now ferromagnetic. In the longitudinal direction, on the other hand, the correlations remain weakly antiferromagnetic. The peak at $(\pi, \pi)$, however, is now slightly split indicating incommensurate correlations.

This transition at half-filling from fully antiferromagnetic correlations to ferromagnetic in-plane and antiferromagnetic out-of-plane correlations with increasing canting angle can be understood as follows: The $t_{2}^{\prime}$ term in the Hamiltonian (2) describes spin-flip hopping and therefore kinematically favors a ferromagnetic alignment of spins. At the same time, the other hopping terms still prefer an antiferromagnetic alignment. As the importance of the $t_{2}^{\prime}$ term increases with increasing canting angle, the system compromises by developing in-plane ferromagnetic correlations whereas the out-of-plane correlations, while weakened, remain antiferromagnetic.

Next, we investigate the changes to the magnetic structure when doping the system. Here we keep $\theta=20^{\circ}$ fixed and consider both the electron and the hole doped case. For the electron doped case, $\langle n\rangle=2.8$, shown in the fourth column of Fig. 4, we find that the longitudinal correlation function now has a peak at $(0, \pi)$, i.e., the correlations are ferromagnetic along the legs but antiferromagnetic between the two legs. The transverse correlations appear to be largely incommensurate. In contrast, the hole doped case $\langle n\rangle=1.2$ shown in the last column of Fig. 4 has dominant $(\pi, 0)$ correlations: antiferromagnetic along the legs and ferromagnetic between the two legs, both in- and out-of-plane. In both cases the peaks are less well-defined than in the undoped case and the magnetic order is much weaker. 
Clearly, all these results are affected by the small cluster sizes and related boundary effects. Nevertheless, there are two main conclusions we can draw in the doped case: (1) the results are strongly particle-hole asymmetric, and (2) doping does weaken the magnetic correlations and also tends to push them to become more incommensurate with the lattice.

In conclusion, we have gained important qualitative insights into the model Hamiltonian (2) for intermediate interaction strengths. At half-filling, the antiferromagnetic order is weakened with increasing canting angle, ultimately leading to a transition to ferromagnetic in-plane correlations for $\theta \gtrsim 20^{\circ}$. Doping the system further weakens the magnetic order while making the correlations also more incommensurate. As expected, the cases of hole and electron doping are not equivalent. Based on the exact diagonalizations of such small clusters, we cannot make any statements about how long-range these magnetic structures are and what happens if such a basic building block is coupled to its surroundings. To address these questions, we calculate the magnetic response for the full two-dimensional lattice using the random-phase approximation next.

\section{RANDOM-PHASE APPROXIMATION}

In the single-particle response, the effects of correlations weaken rapidly with doping, such that one may expect the random phase approximation to provide an adequate description of the two-particle response. However, this is not necessarily true as has been shown for the case of the cuprates in Ref. [65]. Since we are here interested in a much more moderately correlated regime as compared to the cuprates we might, however, nevertheless expect that RPA provides a good starting point to analyze the basic physical properties of this model.

\section{A. Response functions}

In the framework of linear response theory and using the Kubo formula, the physical components of the bare susceptibilities are given by [66]

$$
\begin{aligned}
& \chi_{0}^{u v}\left(\mathbf{q}, \mathrm{i} \omega_{n}\right)= \\
& -\frac{T}{4 N} \sum_{\mathbf{k}, \mathrm{i} \nu_{m}} \operatorname{Tr}\left[\check{\boldsymbol{\sigma}}^{u} \check{G}_{0}\left(\mathbf{k}, \mathrm{i} \nu_{m}\right) \check{\boldsymbol{\sigma}}^{v} \check{G}_{0}\left(\mathbf{k}+\mathbf{q}, \mathrm{i} \nu_{m}+\mathrm{i} \omega_{n}\right)\right]
\end{aligned}
$$

in which $u, v=0$, and $u, v=\{x, y, z\}$ are denoting the charge and spin components of the bare susceptibility. Moreover, the trace is performed over sublattice and spin spaces and

$$
\check{\boldsymbol{\sigma}}^{u}= \begin{cases}\mathbb{1} & u=0 \\ \tau^{z} \otimes \sigma^{u} & u \neq 0\end{cases}
$$

where $\mathbb{1}$ is a $4 \times 4$ unit matrix and $\hat{\tau}=\left(\tau^{x}, \tau^{y}, \tau^{z}\right)$ are Pauli matrices in sublattice space. Besides, the unperturbed electron
Green's function in the same basis is defined as

$$
\check{G}_{0}\left(\mathbf{k}, \mathrm{i} \nu_{m}\right)=\left[\mathrm{i} \nu_{m} \mathbb{1}-\mathcal{H}_{0}(\mathbf{k})\right]^{-1}
$$

where $\nu_{m}$ are Matsubara frequencies. One can write the unperturbed Green's function matrix as

$$
\check{G}_{0}\left(\mathbf{k}, \mathrm{i} \nu_{m}\right)=\left[\begin{array}{ll}
\hat{G}_{0}^{\mathrm{AA}}\left(\mathbf{k}, \mathrm{i} \nu_{m}\right) & \hat{G}_{0}^{\mathrm{AB}}\left(\mathbf{k}, \mathrm{i} \nu_{m}\right) \\
\hat{G}_{0}^{\mathrm{BA}}\left(\mathbf{k}, \mathrm{i} \nu_{m}\right) & \hat{G}_{0}^{\mathrm{BB}}\left(\mathbf{k}, \mathrm{i} \nu_{m}\right)
\end{array}\right] .
$$

Then, the bare susceptibility within the sublattice-spin basis can be rewritten as

$$
\begin{aligned}
& \chi_{0}^{u v}\left(\mathbf{q}, \mathrm{i} \omega_{n}\right)=-\frac{T}{4 N} \sum_{\mathbf{k}, \mathrm{i} \nu_{m}} \sum_{p p^{\prime} p^{\prime \prime}} \\
& \operatorname{Tr}_{\sigma}\left[\hat{\sigma}^{u} \hat{G}_{0}^{p p^{\prime}}\left(\mathbf{k}, \mathrm{i} \nu_{m}\right) \hat{\sigma}^{v} \hat{G}_{0}^{p^{\prime} p^{\prime \prime}}\left(\mathbf{k}+\mathbf{q}, \mathrm{i} \nu_{m}+\mathrm{i} \omega_{n}\right)\right] .
\end{aligned}
$$

The transformation of the free electron Green's function from the sublattice-spin into the band pseudospin basis is achieved by

$$
G_{0, \sigma \sigma^{\prime}}^{p p^{\prime}}\left(\mathbf{k}, \mathrm{i} \nu_{m}\right)=\sum_{s} \Lambda_{p \sigma}^{s}(\mathbf{k}) \Lambda_{p^{\prime} \sigma^{\prime}}^{* s}(\mathbf{k}) G_{0}^{s}\left(\mathbf{k}, \mathrm{i} \nu_{m}\right),
$$

where the number of bands in Eq. (8) is represented by $s=$ $1,2,3,4$, and $\Lambda_{p \sigma}^{s}(\mathbf{k})=\langle\mathbf{k}, p \sigma \mid \mathbf{k}, s\rangle$ denotes the matrix elements to connect the $s$-th band to sublattice $p(=\mathrm{A}$ or B) and pseudospin $\sigma$. Hence, the spatial components of the bare susceptibility are given by

$$
\begin{aligned}
& \chi_{0}^{u v}\left(\mathbf{q}, \mathrm{i} \omega_{n}\right)= \\
& -\frac{T}{4 N} \sum_{\mathbf{k}, s s^{\prime}, \mathrm{i} \nu_{m}} \zeta_{u v}^{s s^{\prime}}(\mathbf{k}, \mathbf{q}) G_{0}^{s}\left(\mathbf{k}, \mathrm{i} \nu_{m}\right) G_{0}^{s^{\prime}}\left(\mathbf{k}+\mathbf{q}, \mathrm{i} \nu_{m}+\mathrm{i} \omega_{n}\right),
\end{aligned}
$$

with

$$
\begin{aligned}
& \zeta_{u v}^{s s^{\prime}}(\mathbf{k}, \mathbf{q})= \\
& \Lambda_{p \sigma}^{* s^{\prime}}(\mathbf{k}+\mathbf{q}) \sigma_{\sigma \sigma^{\prime}}^{u} \Lambda_{p^{\prime} \sigma^{\prime}}^{s}(\mathbf{k}) \Lambda_{p^{\prime} \delta}^{s^{\prime}}(\mathbf{k}+\mathbf{q}) \sigma_{\delta^{\prime} \delta}^{v} \Lambda_{p^{\prime \prime} \delta^{\prime}}^{* s}(\mathbf{k})
\end{aligned}
$$

where a summation is performed over the repeated spinindices. If we now sum over the fermionic Matsubara frequency $\mathrm{i} \nu_{m}$ and do an analytical continuation $\mathrm{i} \omega_{n} \rightarrow \omega+\mathrm{i} 0^{+}$ then we obtain the well-known Lindhard function for the retarded bare susceptibility

$$
\chi_{0}^{u v}(\mathbf{q}, \omega)=\frac{1}{4 N} \sum_{\mathbf{k}, s s^{\prime}} \zeta_{u v}^{s s^{\prime}}(\mathbf{k}, \mathbf{q}) \frac{f\left(E_{\mathbf{k}+\mathbf{q}}^{s^{\prime}}\right)-f\left(E_{\mathbf{k}}^{s}\right)}{E_{\mathbf{k}}^{s}-E_{\mathbf{k}+\mathbf{q}}^{s^{\prime}}+\omega+\mathrm{i} 0^{+}} .
$$

Within RPA, the matrix of susceptibilities is then given by

$$
\hat{\chi}_{\mathrm{RPA}}(\mathbf{q}, \omega)=\frac{1}{1-\hat{U}(\mathbf{q}) \hat{\chi}_{0}(\mathbf{q}, \omega)} \hat{\chi}_{0}(\mathbf{q}, \omega),
$$


where $\hat{U}(\mathbf{q})$ denotes the bare interaction matrix with

$$
\hat{U}(\mathbf{q})=\sum_{u v} \delta_{u, v}\left[\delta_{u, 0} V(\mathbf{q})-(-1)^{\delta_{u, 0}} \frac{U}{8}\right] .
$$

In this equation, $\delta_{u, v}$ is the Kronecker delta and $V(\mathbf{q})=$ $2 V\left(\cos q_{x}+\cos q_{y}\right)$. In the spin (charge) channel at a specific value of $U=U_{c}\left(V=V_{c}\right)$, the determinant of the denominator of Eq. (18) vanishes, $\left|1-\hat{U}(\mathbf{q}) \hat{\chi}_{0}(\mathbf{q}, \omega)\right|=0$, generating an instability towards an ordered spin-density wave (chargedensity wave) state [67].

In addition to the zero-frequency spin and charge susceptibilities, we are also calculating the charge-charge and spinspin two-point correlation functions which are obtained by the Fourier transformation of the charge and spin susceptibility, respectively. The density-density correlation function is expressed as

$$
\langle n(\mathbf{0}) n(\mathbf{r})\rangle=\frac{1}{4 N} \sum_{\mathbf{q}} e^{\mathrm{i} \mathbf{q} \cdot \mathbf{r}} \chi_{\mathrm{RPA}}^{00}(\mathbf{q}, \omega=0) .
$$

Furthermore, the in-plane and out-of-plane components of spin-spin correlation function are given by

$$
\begin{aligned}
\left\langle S^{+}(\mathbf{0}) S^{-}(\mathbf{r})\right\rangle & =\frac{1}{4 N} \sum_{\mathbf{q}} e^{\mathrm{iq} \cdot \mathbf{r}} \chi_{\mathrm{RPA}}^{+-}(\mathbf{q}, \omega=0), \\
\left\langle S^{z}(\mathbf{0}) S^{z}(\mathbf{r})\right\rangle & =\frac{1}{4 N} \sum_{\mathbf{q}} e^{\mathrm{iq} \cdot \mathbf{r}} \chi_{\mathrm{RPA}}^{z z}(\mathbf{q}, \omega=0),
\end{aligned}
$$

where

$$
\begin{aligned}
& \chi_{\mathrm{RPA}}^{+-}(\mathbf{q}, \omega)= \\
& \chi_{\mathrm{RPA}}^{x x}(\mathbf{q}, \omega)+\chi_{\mathrm{RPA}}^{y y}(\mathbf{q}, \omega)-\mathrm{i}\left[\chi_{\mathrm{RPA}}^{x y}(\mathbf{q}, \omega)-\chi_{\mathrm{RPA}}^{y x}(\mathbf{q}, \omega)\right]
\end{aligned}
$$

describes the in-plane component of the spin susceptibility corresponding to spin flipping processes.

We also consider the dynamical structure factor $S_{\mathrm{RPA}}^{u v}(\mathbf{q}, \omega)$. Technically, we follow earlier studies $[68,69]$ for calculating the different branches of spin excitations within RPA and use the formula

$$
S_{\mathrm{RPA}}^{u v}(\mathbf{q}, \omega)=-2 \operatorname{Im}\left[\chi_{\mathrm{RPA}}^{u v}(\mathbf{q}, \omega)\right] .
$$

\section{B. Results}

We start by presenting and discussing our main result, the magnetic phase diagram as a function of doping and canting angle, shown in Fig. 5. We note first that in the density of states (DOS) at the Fermi level there is an asymmetry in the position of the van-Hove filling $\langle n\rangle_{\mathrm{vH}}$ due to the non-zero next-nearest neighbor hopping, see Fig. 5(a). By increasing the canting angle, the van-Hove filling approaches half filling, $\langle n\rangle=2$. The position of the van-Hove singularities can help us to understand the transitions in the nature of the magnetic fluctuations [40]. Fig. 5(b) shows the magnetic phase diagram of the system for $U=1$ obtained within RPA. Since the extended Hubbard term is expected to be small and will

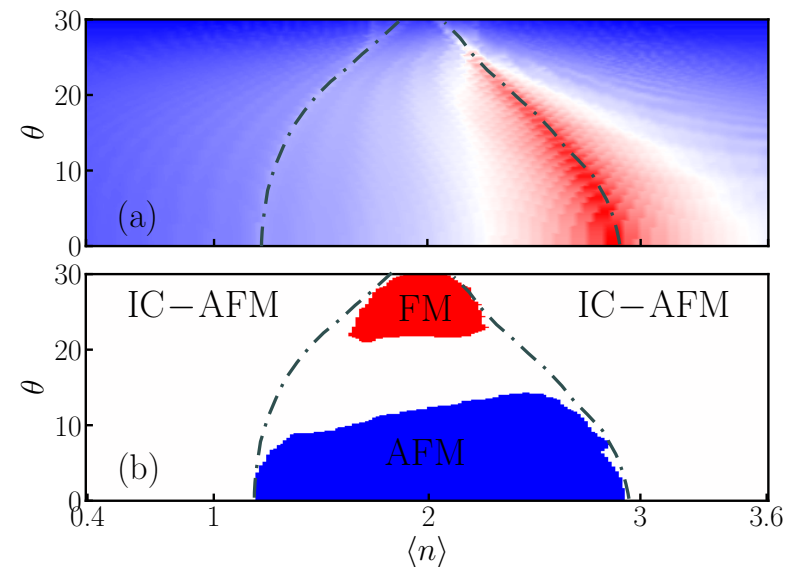

FIG. 5. (a) Density of states at the Fermi surface $(\omega=0)$ of the non-interacting model versus filling and canting angle. The lines denote the position of the van-Hove singularities for specific values of filling, $\langle n\rangle_{\mathrm{vH}}$, and distortions of the Ir-O-Ir bonds. (b) Magnetic phase diagram obtained by RPA as a function of filling and canting angle for $U=1$ and $V=0$. The lines denote again the position of the van-Hove singularities.
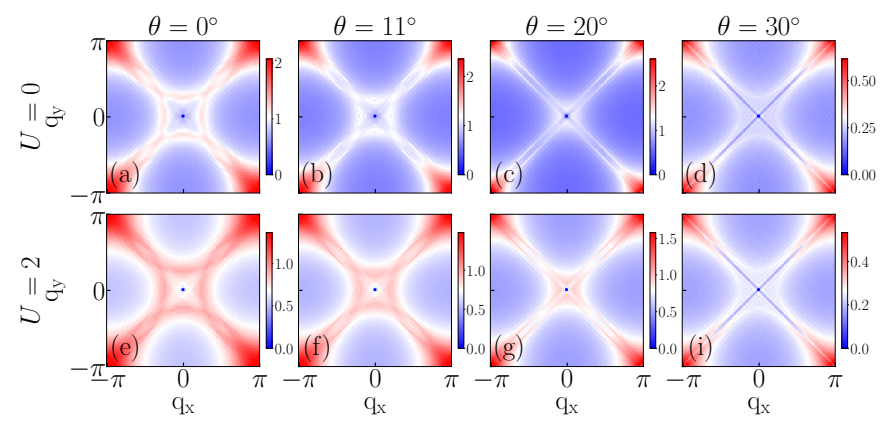

FIG. 6. Momentum dependence of the zero-frequency charge susceptibility $\chi^{00}(\mathbf{q}, \omega=0)$ for $\langle n\rangle=2.4$ and different values of the canting angle. The top row shows the bare susceptibilities, and the bottom the corresponding RPA susceptibilities for $U=2$. We set $V=0$. The values of the RPA charge susceptibilities remain positive, i.e. the system is far from a CDW instability.

thus only affect the charge fluctuations, we set $V=0$ for now and return to the effects of a finite $V$ later. At low levels of canting angle, near $\theta=0^{\circ}$, there are two distinguishable van-Hove fillings near $\langle n\rangle_{\mathrm{vH}, 1} \approx 1$ and $\langle n\rangle_{\mathrm{vH}, 2} \approx 3$. The interval $\langle n\rangle_{\mathrm{vH}, 1} \lesssim\langle n\rangle \lesssim\langle n\rangle_{\mathrm{vH}, 2}$ includes the area of commensurate antiferromagnetic (AFM) fluctuations in the system. Outside of this region, incommensurate AFM (IC-AFM) fluctuations dominate. Increasing the canting angle reduces the region in doping with commensurate AFM fluctuations. This can be explained by the van-Hove singularities moving towards half filling. Finally, near half filling and for canting angles $\theta \gtrsim 20^{\circ}$, ferromagnetic fluctuations are established. Note that these fluctuations are not necessarily long ranged as we will discuss in detail in the following.

In Fig. 6, the evolution of the zero-frequency charge susceptibility $\chi^{00}(\mathbf{q}, \omega=0)$ is shown in the electron doped case as a function of canting angle. As might be expected, we find that the on-site Hubbard interaction has little effect; the 

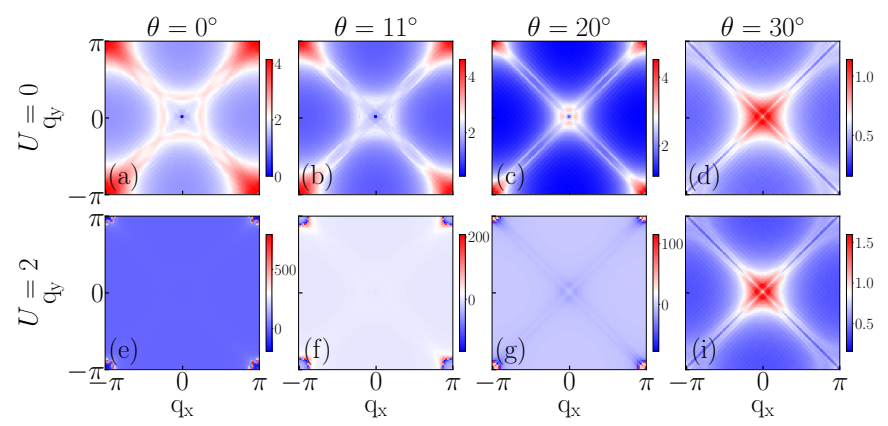

FIG. 7. Momentum dependence of the in-plane component of the zero-frequency spin susceptibility $\chi^{+-}(\mathbf{q}, \omega=0)$ for $\langle n\rangle=2.4$ and different values of $\theta$. The panels in the top row show the bare susceptibilities while the corresponding RPA results are presented in the bottom row. We have set $V=0$.
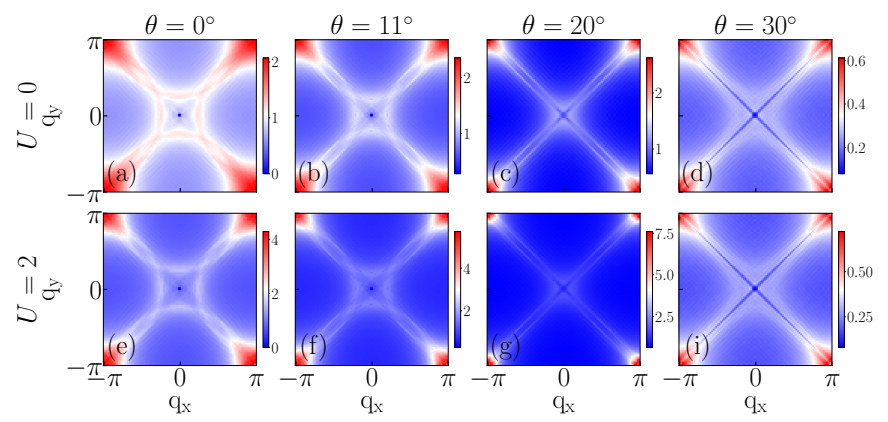

FIG. 8. Same as Fig. 7 but for the out-of-plane spin susceptibility $\chi^{z z}(\mathbf{q}, \omega=0)$.

data shown in the bottom row of Fig. 6 are representative for $U \leq 3$. Charge fluctuations with $\mathbf{q} \sim(\pi, \pi)$ are dominant but remain always short-ranged-we are far from a CDW instability.

The momentum structure of the zero-frequency in-plane spin susceptibility as a function of $U$ and $\theta$ is presented in Fig. 7. The bare susceptibilities $\chi_{0}^{+-}(\mathbf{q}, \omega=0)$, shown in Figs. 7(a)-(d), display nearly commensurate AFM fluctuations, $\mathbf{q} \sim(\pi, \pi)$, for $\theta=0^{\circ}, 11^{\circ}, 20^{\circ}$ but nearly ferromagnetic fluctuations for $\theta=30^{\circ}$. These fluctuations are, however, all short ranged. For $U=2$, the RPA susceptibilities for $\theta=0^{\circ}, 11^{\circ}, 20^{\circ}$ become negative, indicating that long-range antiferromagnetic has been established. For $\theta=30^{\circ}$ the RPA susceptibility is then again very similar to the bare one: the ferromagnetic fluctuations remain short ranged.

In Fig. 8 we show the corresponding out-of-plane spin susceptibilities. Here we find that for all canting angles commensurate, short-ranged antiferromagnetic fluctuations are present. The Hubbard interaction has, in this case, only a small effect. The RPA susceptibilities are very similar to the bare ones.

To summarize these findings for the electron-doped case, we show in Fig. 9 the zero-frequency two-point spin and charge correlation functions along the crystallographic $a$-axis. The out-of-plane spin correlations $\left\langle S^{z}(\mathbf{0}) S^{z}(\mathbf{r})\right\rangle$ are always short-ranged and close to commensurate irrespective of the
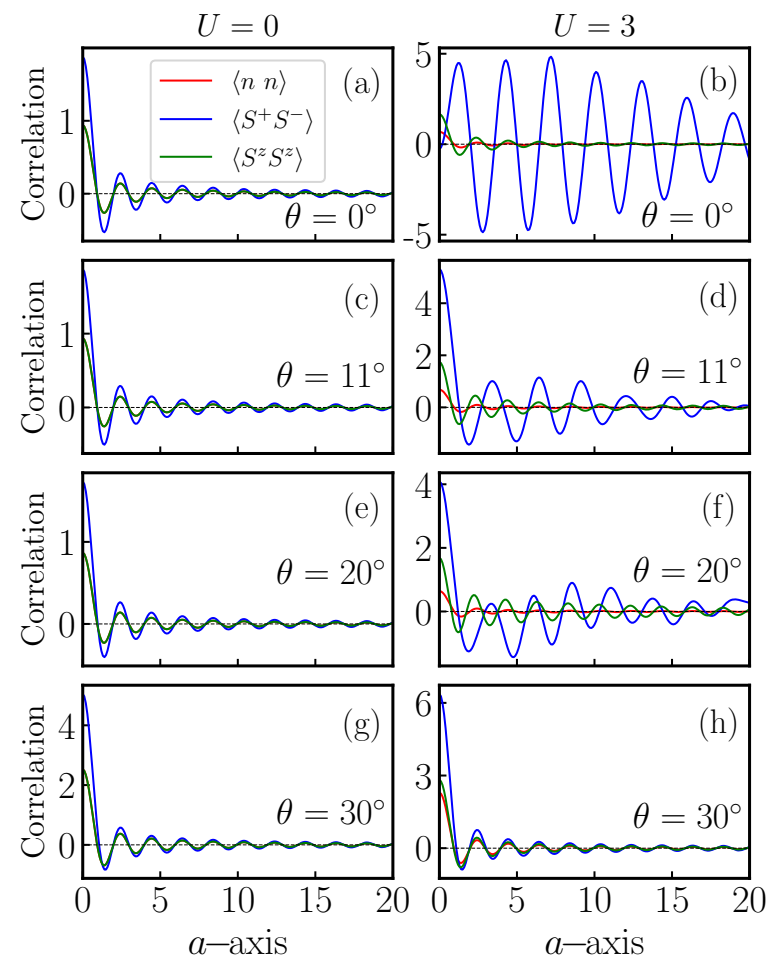

FIG. 9. Charge-charge (red), in-plane spin-spin (blue), and outof-plane spin-spin (green) two-point correlation functions along the crystallographic $a$-axis for the electron-doped case with $\langle n\rangle=2.4$. The left column shows the non-interacting case and the right column the case with $U=3$ for different canting angles $\theta$. We set $V=0$.

Hubbard interaction or the canting angle. For the in-plane correlations $\left\langle S^{+}(\mathbf{0}) S^{-}(\mathbf{r})\right\rangle$ we do see, on the other hand, large changes as a function of the Hubbard interaction $U$ and the canting angle $\theta$. For $\theta=0^{\circ}, 11^{\circ}, 20^{\circ}$ the Hubbard interaction establishes long-range antiferromagnetic correlations in the system. These correlations are fully commensurate for $\theta=0^{\circ}$ and become slightly incommensurate for $\theta=11^{\circ}, 20^{\circ}$. For all canting angles with and without interactions the chargecharge correlations remain always commensurate and short ranged. For pure on-site Hubbard interactions there is no CDW instability.

This changes, however, if we also allow for a moderate nearest-neighbor interaction $V$ as is shown in Fig. 10. Here we have set $U=0$ to concentrate on the effects of $V$. In contrast to Fig. 6, where even moderately strong Hubbard interactions $U$ had very little effect on the charge susceptibility, the nearest-neighbor interaction dramatically changes the charge response even for strengths as small as $V=0.2$. For $\theta=0^{\circ}, 11^{\circ}$ the response is strongly enhanced as compared to the non-interacting case and for $\theta=20^{\circ}$ there is an instability towards long-range CDW order. Increasing $V$ further we find a CDW instability for all $\theta \lesssim 20^{\circ}$. For even larger canting angles, on the other hand, there is no charge order as can be seen from the last column of Fig. 10. We conclude, that even relatively small nearest-neighbor Coulomb interactions can lead to CDW instabilities in addition to the magnetic instabilities discussed earlier. For general $U$ and $V$, we expect a competition between these different types of orders. 

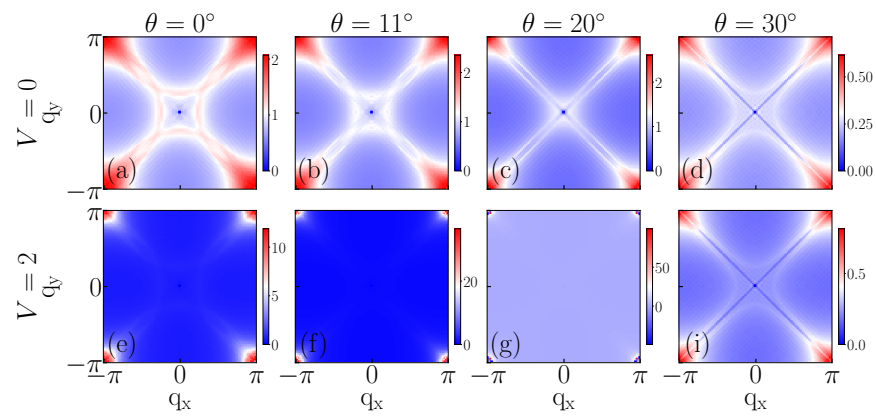

FIG. 10. Charge susceptibility $\chi^{00}(\mathbf{q}, \omega=0)$ for the same parameters as shown in Fig. 6 but now we set $U=0$ and compare the noninteracting case with the case of weak nearest-neighbor Coulomb interactions, $V=0.2$.

So far, we have concentrated on investigating the electrondoped case because of its similarities to the hole-doped cuprates. Motivated by a recent resonant inelastic X-ray scattering (RIXS) study of the magnetic excitations in $\mathrm{Sr}_{2} \mathrm{IrO}_{4}$ in the hole-doped regime, we have used Eq. (23) to obtain the dynamical spin structure factors-both in-plane and out-ofplane-within RPA. The results are shown in Fig. 11. We find several branches of magnetic excitations with gapless points at $\mathbf{q}=(0,0)$ and $\mathbf{q}=(\pi, \pi)$. The overall structure of the magnetic excitations at low energies is in good qualitative agreement with the experimental results reported in Ref. [70]. We note, however, that we also find other branches of magnetic excitations between $\mathbf{q}=(0,0)$ and $\mathbf{q}=(\pi, \pi)$ at much higher energies which have not been observed experimentally. Since we have not taken into account the experimental resolution nor the atomic form factors, our results are meant merely as a qualitative check. A more detailed analysis would require to reduce our model to an effective Heisenberg or t-J model. The experimental results in Ref. [70] also suggest that it might be important to also include third neighbor hopping to accurately describe the dispersions across the full Brillouin zone, hopping processes which we have neglected here.

\section{CONCLUSIONS}

We have studied a two-dimensional single band Hubbard model with asymmetric spin-orbit couplings which is a minimal model for the $5 d$ layered transition metal oxides such as $\mathrm{Sr}_{2} \mathrm{IrO}_{4}$. Most of our study has been focused on the electrondoped case which is believed to show similarities to the holedoped cuprates. As a first step, we have investigated the electronic band structure of the non-interacting system as a function of the Ir-O-Ir bond angle. With increasing bond angle, the spin-dependent hopping terms become more important leading to a small splitting of the bands. At the same time, the second nearest-neighbor hopping amplitude decreases and the dispersions become almost particle-hole symmetric for large canting angles $\theta \sim 30^{\circ}$. The almost flat bands which form in the latter case between high-symmetry points in the Brillouin zone are an interesting aspect of this model which we

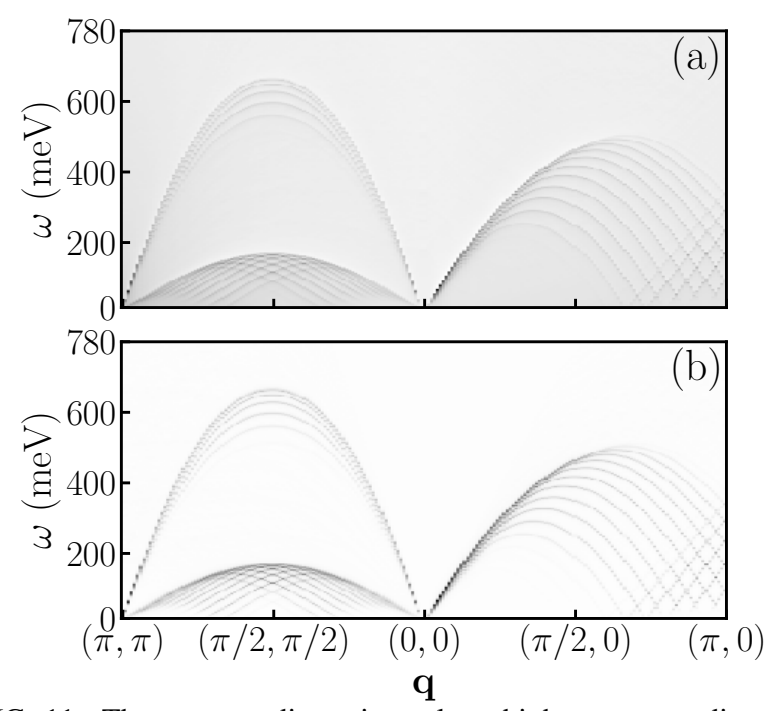

FIG. 11. The magnon dispersions along high-symmetry directions for the hole-doped case with $\langle n\rangle=1.6, U=2$, and $V=0$ obtained in RPA. Panel (a) shows the in-plane magnetic excitations while panel (b) shows the out-of-plane contributions.

plan to further explore in future studies of the superconducting and possibly topological properties of this model. To understand the effects of on-site and nearest-neighbor interactions, we have used exact diagonalizations of small clusters and the random-phase approximation. From these calculations a consistent picture of the magnetic instabilities of the system emerges: For the electron doped case with $\langle n\rangle=2.4, U \gtrsim 2$ and canting angles $\theta \lesssim 15^{\circ}$ long-range, in-plane antiferromagnetic order forms. This order is replaced by dominant ferromagnetic fluctuations for larger canting angles. More generally speaking, we have been able to map out the regions of the $(U, \theta)$ phase diagram where antiferromagnetic or ferromagnetic fluctuations dominate. We have found that the borders of these commensurate magnetic regions around halffilling are defined by van-Hove singularities with incommensurate fluctuations dominating for larger doping levels. Furthermore, we have shown that fairly modest nearest-neighbor Hubbard interactions can give rise to additional CDW instabilities. The model thus shows an intricate interplay between various magnetic and charge instabilities as a function of the canting angle-which controls the strength of the spin-orbit coupling and of the next-nearest neighbor hopping amplitudeand the on-site and nearest-neighbor Hubbard interactions $U$ and $V$.

While most of our study has focussed on the electron-doped case, we have also studied the magnetic excitations on the hole-doped side. We find that the spin-structure factor has gapless points at $\mathbf{q}=(0,0)$ and $\mathbf{q}=(\pi, \pi)$. The magnon dispersions which we obtain in our model calculations are qualitatively consistent with recent RIXS experiments on holedoped $\mathrm{Sr}_{2} \mathrm{IrO}_{4}$. While a more accurate description of the experimentally observed dispersions might require to also include third neighbor hopping processes, as has been in suggested in Ref. [70], the minimal model considered here does already give the right energy scales and overall shape of the 
dispersions.

For the future it would be interesting to see whether the model does support superconductivity and if so, what the dominant superconducting channels are. This might help to further clarify the similarities and differences between the electron-doped iridates and the hole-doped cuprates.

\section{ACKNOWLEDGMENTS}

We are grateful to Y. Yanase, G. Jackeli, B.J. Kim, I. Eremin, P. Thalmeier, G. Khaliullin, D.K. Singh and
Y. Bang for fruitful discussions. M. B. acknowledges S. A. Jafari, M. H. Zare, and R. Jafari for fruitful discussions. A. A. acknowledges the support of the Max PlanckPOSTECH-Hsinchu Center for Complex Phase Materials, and financial support from the National Research Foundation (NRF) funded by the Ministry of Science of Korea (Grant No. 2016K1A4A01922028). J. S. acknowledges support by the Natural Sciences and Engineering Research Council (NSERC, Canada) and by the Deutsche Forschungsgemeinschaft (DFG) via Research Unit FOR 2316.
[1] E. Dagotto and T. M. Rice, Science 271, 618 (1996).

[2] Y. Tokura and N. Nagaosa, Science 288, 462 (2000).

[3] E. Dagotto, Science 309, 257 (2005).

[4] P. A. Lee, N. Nagaosa, and X.-G. Wen, Rev. Mod. Phys. 78, 17 (2006).

[5] B. Lake, A. M. Tsvelik, S. Notbohm, D. Alan Tennant, T. G. Perring, M. Reehuis, C. Sekar, G. Krabbes, and B. Büchner, Nature Physics 6, 50 (2010).

[6] D. J. Scalapino, Rev. Mod. Phys. 84, 1383 (2012).

[7] S. K. Choi, R. Coldea, A. N. Kolmogorov, T. Lancaster, I. I. Mazin, S. J. Blundell, P. G. Radaelli, Y. Singh, P. Gegenwart, K. R. Choi, S.-W. Cheong, P. J. Baker, C. Stock, and J. Taylor, Phys. Rev. Lett. 108, 127204 (2012).

[8] S. H. Chun, J.-W. Kim, J. Kim, H. Zheng, C. C. Stoumpos, C. D. Malliakas, J. F. Mitchell, K. Mehlawat, Y. Singh, Y. Choi, T. Gog, A. Al-Zein, M. Moretti Sala, M. Krisch, J. Chaloupka, G. Jackeli, G. Khaliullin, and B. J. Kim, arXiv e-prints , arXiv:1504.03618 (2015), 1504.03618 [cond-mat.mtrl-sci].

[9] Q. Cui, J.-G. Cheng, W. Fan, A. E. Taylor, S. Calder, M. A. McGuire, J.-Q. Yan, D. Meyers, X. Li, Y. Q. Cai, Y. Y. Jiao, Y. Choi, D. Haskel, H. Gotou, Y. Uwatoko, J. Chakhalian, A. D. Christianson, S. Yunoki, J. B. Goodenough, and J.-S. Zhou, Phys. Rev. Lett. 117, 176603 (2016).

[10] M. Majumder, R. S. Manna, G. Simutis, J. C. Orain, T. Dey, F. Freund, A. Jesche, R. Khasanov, P. K. Biswas, E. Bykova, N. Dubrovinskaia, L. S. Dubrovinsky, R. Yadav, L. Hozoi, S. Nishimoto, A. A. Tsirlin, and P. Gegenwart, Phys. Rev. Lett. 120, 237202 (2018).

[11] K. Kitagawa, T. Takayama, Y. Matsumoto, A. Kato, R. Takano, Y. Kishimoto, S. Bette, R. Dinnebier, G. Jackeli, and H. Takagi, Nature 554, 341 (2018).

[12] J. Bertinshaw, Y. Kim, G. Khaliullin, and B. Kim, Annual Review of Condensed Matter Physics 10, 315 (2019).

[13] R.-Y. Sun, Z. Zhu, and Z.-Y. Weng, Phys. Rev. Research 2, 033007 (2020).

[14] J. M. Tranquada, B. J. Sternlieb, J. D. Axe, Y. Nakamura, and S. Uchida, Nature (London) 375, 561 (1995).

[15] H. A. Mook, P. Dai, and F. Doğan, Phys. Rev. Lett. 88, 097004 (2002).

[16] R. Kajimoto, K. Ishizaka, H. Yoshizawa, and Y. Tokura, Phys. Rev. B 67, 014511 (2003).

[17] C. Howald, H. Eisaki, N. Kaneko, M. Greven, and A. Kapitulnik, Phys. Rev. B 67, 014533 (2003).

[18] X. Chen, J. L. Schmehr, Z. Islam, Z. Porter, E. Zoghlin, K. Finkelstein, J. P. C. Ruff, and S. D. Wilson, Nature Communications 9, 103 (2018).
[19] D. Pesin and L. Balents, Nature Physics 6, 376 (2010).

[20] W. Witczak-Krempa, G. Chen, Y. B. Kim, and L. Balents, Annual Review of Condensed Matter Physics 5, 57 (2014).

[21] J. G. Rau, E. K.-H. Lee, and H.-Y. Kee, Annual Review of Condensed Matter Physics 7, 195 (2016).

[22] S. Fujiyama, H. Ohsumi, T. Komesu, J. Matsuno, B. J. Kim, M. Takata, T. Arima, and H. Takagi, Phys. Rev. Lett. 108, 247212 (2012).

[23] F. Wang and T. Senthil, Phys. Rev. Lett. 106, 136402 (2011).

[24] Y. K. Kim, O. Krupin, J. D. Denlinger, A. Bostwick, E. Rotenberg, Q. Zhao, J. F. Mitchell, J. W. Allen, and B. J. Kim, Science 345, 187 (2014).

[25] H. Watanabe, T. Shirakawa, and S. Yunoki, Phys. Rev. Lett. 110, 027002 (2013).

[26] Z. Y. Meng, Y. B. Kim, and H.-Y. Kee, Phys. Rev. Lett. 113, 177003 (2014).

[27] A. de la Torre, S. McKeown Walker, F. Y. Bruno, S. Riccó, Z. Wang, I. Gutierrez Lezama, G. Scheerer, G. Giriat, D. Jaccard, C. Berthod, T. K. Kim, M. Hoesch, E. C. Hunter, R. S. Perry, A. Tamai, and F. Baumberger, Phys. Rev. Lett. 115, 176402 (2015).

[28] M.-H. Zare, M. Biderang, and A. Akbari, Phys. Rev. B 96, 205156 (2017).

[29] J. Nelson, C. Parzyck, B. Faeth, J. Kawasaki, D. Schlom, and M. Shen, Nature Communications 11 (2020), 10.1038/s41467020-16425-z.

[30] L. P. Gor'kov and E. I. Rashba, Phys. Rev. Lett. 87, 037004 (2001).

[31] E. Bauer, G. Hilscher, H. Michor, C. Paul, E. W. Scheidt, A. Gribanov, Y. Seropegin, H. Noël, M. Sigrist, and P. Rogl, Phys. Rev. Lett. 92, 027003 (2004).

[32] M. Yogi, Y. Kitaoka, S. Hashimoto, T. Yasuda, R. Settai, T. D. Matsuda, Y. Haga, Y. Ōnuki, P. Rogl, and E. Bauer, Phys. Rev. Lett. 93, 027003 (2004).

[33] K. V. Samokhin, E. S. Zijlstra, and S. K. Bose, Phys. Rev. B 69, 094514 (2004).

[34] P. A. Frigeri, D. F. Agterberg, A. Koga, and M. Sigrist, Phys. Rev. Lett. 92, 097001 (2004).

[35] S. Fujimoto, Journal of the Physical Society of Japan 76, 051008 (2007).

[36] Y. Yanase, Journal of the Physical Society of Japan 79, 084701 (2010).

[37] E. Bauer and M. Sigrist, Non-Centrosymmetric Superconductors: Introduction and Overview (Springer Berlin Heidelberg, 2012). 
[38] M. Biderang, H. Yavari, M.-H. Zare, P. Thalmeier, and A. Akbari, Phys. Rev. B 98, 014524 (2018).

[39] A. Greco and A. P. Schnyder, Phys. Rev. Lett. 120, 177002 (2018).

[40] A. Greco, M. Bejas, and A. P. Schnyder, Phys. Rev. B 101, 174420 (2020).

[41] X.-L. Qi and S.-C. Zhang, Rev. Mod. Phys. 83, 1057 (2011).

[42] C. W. J. Beenakker, Rev. Mod. Phys. 87, 1037 (2015).

[43] M. H. Fischer, F. Loder, and M. Sigrist, Phys. Rev. B 84, 184533 (2011).

[44] S. Sumita, T. Nomoto, and Y. Yanase, Phys. Rev. Lett. 119, 027001 (2017).

[45] J. Ishizuka and Y. Yanase, Phys. Rev. B 98, 224510 (2018).

[46] H. Shaked, J. Jorgensen, O. Chmaissem, S. Ikeda, and Y. Maeno, Journal of Solid State Chemistry 154, 361 (2000).

[47] M. Subramanian, M. Crawford, R. Harlow, T. Ami, J. Fernandez-Baca, Z. Wang, and D. Johnston, Physica C: Superconductivity 235-240, 743 (1994).

[48] Q. Huang, J. Soubeyroux, O. Chmaissem, I. Sora, A. Santoro, R. Cava, J. Krajewski, and W. Peck, Journal of Solid State Chemistry 112, 355 (1994).

[49] M. K. Crawford, M. A. Subramanian, R. L. Harlow, J. A. Fernandez-Baca, Z. R. Wang, and D. C. Johnston, Phys. Rev. B 49, 9198 (1994).

[50] F. Ye, X. Wang, C. Hoffmann, J. Wang, S. Chi, M. Matsuda, B. C. Chakoumakos, J. A. Fernandez-Baca, and G. Cao, Phys. Rev. B 92, 201112 (2015).

[51] D. H. Torchinsky, H. Chu, L. Zhao, N. B. Perkins, Y. Sizyuk, T. Qi, G. Cao, and D. Hsieh, Phys. Rev. Lett. 114, 096404 (2015).

[52] H. Gretarsson, N. H. Sung, J. Porras, J. Bertinshaw, C. Dietl, J. A. N. Bruin, A. F. Bangura, Y. K. Kim, R. Dinnebier, J. Kim, A. Al-Zein, M. Moretti Sala, M. Krisch, M. Le Tacon, B. Keimer, and B. J. Kim, Phys. Rev. Lett. 117, 107001 (2016).

[53] X. Liu, M. P. M. Dean, Z. Y. Meng, M. H. Upton, T. Qi, T. Gog, Y. Cao, J. Q. Lin, D. Meyers, H. Ding, G. Cao, and J. P. Hill, Phys. Rev. B 93, 241102 (2016).

[54] D. Pincini, J. G. Vale, C. Donnerer, A. de la Torre, E. C. Hunter, R. Perry, M. Moretti Sala, F. Baumberger, and D. F. McMor- row, Phys. Rev. B 96, 075162 (2017).

[55] S. A. Kivelson, E. Fradkin, and V. J. Emery, Nature (London) 393, 550 (1998).

[56] S. J. Moon, H. Jin, W. S. Choi, J. S. Lee, S. S. A. Seo, J. Yu, G. Cao, T. W. Noh, and Y. S. Lee, Phys. Rev. B 80, 195110 (2009).

[57] F. Ye, S. Chi, B. C. Chakoumakos, J. A. Fernandez-Baca, T. Qi, and G. Cao, Phys. Rev. B 87, 140406 (2013).

[58] D. H. Torchinsky, H. Chu, L. Zhao, N. B. Perkins, Y. Sizyuk, T. Qi, G. Cao, and D. Hsieh, Phys. Rev. Lett. 114, 096404 (2015).

[59] B. J. Kim, H. Jin, S. J. Moon, J.-Y. Kim, B.-G. Park, C. S. Leem, J. Yu, T. W. Noh, C. Kim, S.-J. Oh, J.-H. Park, V. Durairaj, G. Cao, and E. Rotenberg, Phys. Rev. Lett. 101, 076402 (2008).

[60] F. Wang and T. Senthil, Phys. Rev. Lett. 106, 136402 (2011).

[61] H. Jin, H. Jeong, T. Ozaki, and J. Yu, Phys. Rev. B 80, 075112 (2009).

[62] S. M. Young and C. L. Kane, Phys. Rev. Lett. 115, 126803 (2015).

[63] A. Moutenet, A. Georges, and M. Ferrero, Phys. Rev. B 97, 155109 (2018).

[64] A. W. Lindquist and H.-Y. Kee, Phys. Rev. B 100, 054512 (2019).

[65] Y. F. Kung, E. A. Nowadnick, C. J. Jia, S. Johnston, B. Moritz, R. T. Scalettar, and T. P. Devereaux, Phys. Rev. B 92, 195108 (2015).

[66] S. Cobo, F. Ahn, I. Eremin, and A. Akbari, Phys. Rev. B 94, 224507 (2016).

[67] R. Ghadimi, M. Kargarian, and S. A. Jafari, Phys. Rev. B 99, 115122 (2019).

[68] R. G. Pereira, J. Sirker, J. S. Caux, R. Hagemans, J. M. Maillet, S. R. White, and I. Affleck, Journal of Statistical Mechanics: Theory and Experiment 2007, 08022 (2007).

[69] A. Akbari and G. Khaliullin, Phys. Rev. B 90, 035137 (2014).

[70] J. Bertinshaw, J. K. Kim, J. Porras, K. Ueda, N. H. Sung, A. Efimenko, A. Bombardi, J. Kim, B. Keimer, and B. J. Kim, Phys. Rev. B 101, 094428 (2020). 